\title{
Neoadjuvant chemotherapy followed by total pancreatectomy with splenectomy and combined vascular resections after preoperative percutaneous transhepatic portal vein stent placement in locally advanced pancreatic cancer with portal vein total obliteration
}

\author{
Yoonwon Kook ${ }^{1,2}$, Munseok Choi ${ }^{1,2}$, Jung Yup Park ${ }^{2,3}$, Yong Eun Chung ${ }^{2,4}$, \\ Man-Deuk Kim ${ }^{2,4}$, Woo Jung Lee ${ }^{1,2}$, and Chang Moo Kang ${ }^{1,2}$ \\ ${ }^{1}$ Division of HBP Surgery, Department of Surgery, Yonsei University College of Medicine, \\ ${ }^{2}$ Pancreatobiliary Cancer Center, Yonsei Cancer Center, Severance Hospital, \\ ${ }^{3}$ Division of Gastroenterology, Department of Internal Medicine, Yonsei University College of Medicine, \\ ${ }^{4}$ Department of Radiology, Severance Hospital, Yonsei University College of Medicine, Seoul, Korea
}

\begin{abstract}
Pancreatic cancer is one of the most lethal malignant diseases in gastrointestinal system that only about $15-20 \%$ of the patients are potential candidates for resection at diagnostic stage. However, with the advent of neoadjuvant chemotherapy and advancement of surgical skills, patients with locally advanced pancreatic cancer (LAPC), which were deemed initially unresectable, have undergone margin negative radical resection. Here, we present a case of a patient with LAPC who was previously treated with neoadjuvant FOLFIRINOX and underwent pancreaticoduodenectomy combined with vascular resection after preoperative percutaneous transhepatic portal vein stent placement to relieve of portal vein obliteration. The patient recovered without any complication and was discharged on day 8 postoperatively. (Ann Hepatobiliary Pancreat Surg 2020;24:551-556)
\end{abstract}

Key Words: Locally advanced pancreatic cancer; Neoadjuvant chemotherapy; Portal vein stent

\section{INTRODUCTION}

Pancreatic cancer is one of the most lethal malignant diseases in gastrointestinal system. Margin-negative pancreatectomy followed by adjuvant chemotherapy is the most effective modality in treating pancreatic cancer. ${ }^{1-3}$ However, at diagnostic stage, potential candidates for resection are about $15-20 \%$ and the rest are locally advanced or metastatic stage of cancer. Currently, neoadjuvant chemotherapy has been introduced to improve oncologic outcomes by increasing margin-negative resection rates, decreasing lymph node invasion, and providing an early treatment of micro-metastatic disease. ${ }^{4}$ In addition, advancement of surgical technique and perioperative management made radical pancreatectomy with combined venous vascular resection safe and effective in managing ad- vanced pancreatic cancer. ${ }^{5}$

Pancreatic head cancer with portal vein (PV) obliteration has several important clinical issues to consider. First, pancreatic cancer invading portal vein may extend even to the superior mesenteric artery. Therefore, biological behavior is expected to be very aggressive. Second, pancreaticoduodenectomy (PD) with combined superior mesenteric vein-splenic vein-portal vein (SMV-SV-PV) confluence and vascular reconstruction using direct end-toend anastomosis or interposition graft should be prepared. If vascular reconstruction is technically impossible, this condition should be regarded as unresectable, or locally advanced pancreatic cancer. Third, development of secondary collateral vessels to maintain mesentero-hepatic flow may result in massive bleeding during dissection. In addition, sequential disconnection of potential collateral ves-

Received: April 17, 2020; Revised: June 15, 2020; Accepted: June 17, 2020

Corresponding author: Chang Moo Kang

Department of Surgery, Yonsei University College of Medicine, Ludlow Faculty Research Building \#201, 50 Yonsei-ro, Seodaemun-gu, Seoul 03722, Korea

Tel: +82-2-313-8289, Fax: +82-2-2228-2135, E-mail: cmkang@yuhs.ac

Copyright (C) 2020 by The Korean Association of Hepato-Biliary-Pancreatic Surgery

This is an Open Access article distributed under the terms of the Creative Commons Attribution Non-Commercial License (http://creativecommons.org/ censes/by-nc/4.0) which permits unrestricted non-commercial use, distribution, and reproduction in any medium, provided the original work is properly cited. Annals of Hepato-Biliary-Pancreatic Surgery • pISSN: 2508-5778 - eISSN: 2508-5859 
sels during the surgical procedure could result in severe venous congestion and secondary edematous change in small intestines, probably providing adverse impact on subsequent pancreaticojejunostomy (PJ), or hepaticojejunostomy (HJ), and overall postoperative recovery outcomes. Therefore, determining accurate preoperative assessment of tumor extent, and appropriate management to decompress the portal vein pressure is mandatory for safe and effective curative pancreatectomy in pancreatic cancer with PV obliteration.

Here, we present a case of successful radical pancreatectomy after preoperative percutaneous transhepatic portal vein stent (PTPVS) in pancreatic head cancer with obliterated portal vein following neoadjuvant chemotherapy. This is thought to be the first official report in managing pancreatic cancer surgery in Korea using the PTPVS approach. Future perspectives and current literatures are discussed.

\section{CASE}

\section{Case presentation}

A 46-year-old male was admitted the hospital due to jaundice and abdominal discomfort in January, 2019. Blood laboratory results showed elevated AST (120 IU/L; normal: 13.0-34.0 IU/L), ALT (128 IU/L; normal: 5.0$46.0 \mathrm{IU} / \mathrm{L})$, total bilirubin $(7.9 \mathrm{mg} / \mathrm{dl}$; normal: $0.5-1.8 \mathrm{mg} /$ $\mathrm{dl})$, direct bilirubin $(6.2 \mathrm{mg} / \mathrm{dl}$; normal: $0.1-0.4 \mathrm{mg} / \mathrm{dl})$, and gamma-GT (1591 IU/L; normal: 12.0-54.0 IU/L). Tumor markers CEA (24.8 ng/ml; normal: 0-5 ng/ml) and CA19-9 (3132.0 U/ml; normal: 0-34 U/ml) were both elevated. Radiologic evaluation (CT scan) showed a pancreatic head cancer $3.5 \mathrm{~cm}$ in size, invading the common bile duct (CBD) and duodenum with biliary dilatation, and also invading the gastroduodenal artery (GDA), portal vein (PV), and superior mesenteric vein (SMV) with encasing the SMV. Endoscopic ultrasonography (EUS) found a $4 \mathrm{~cm}-$
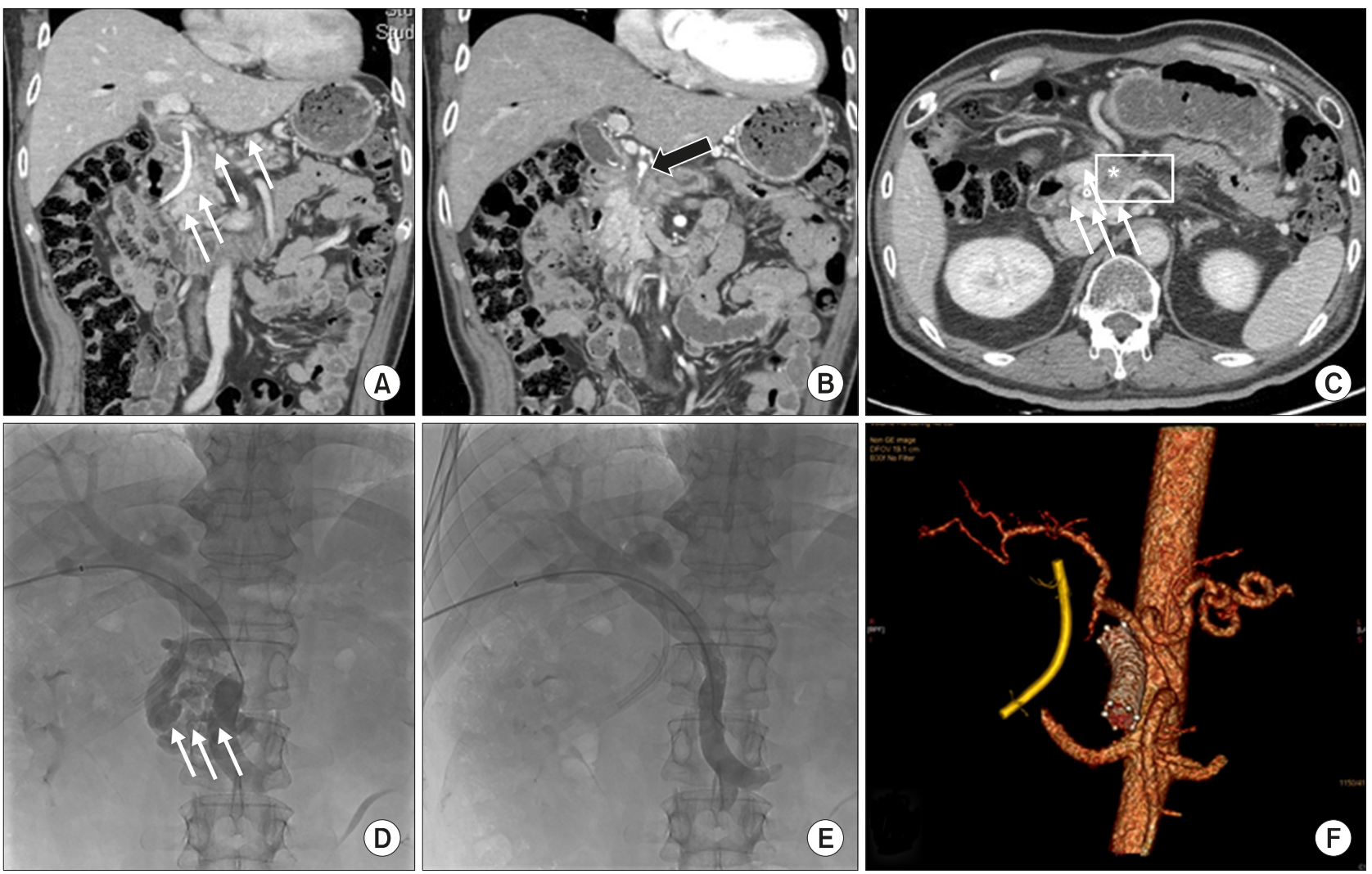

Fig. 1. Preoperative image study and PTPVS insertions. Endoscopic biliary drainage tube and periportal cavernous transformation (thin white arrows) due to total obliteration of portal vein by the tumor $\left(^{*}\right)$ are noted (A and C). Common hepatic artery and gastroduodenal artery invasion is highly suspected (thick black arrow, B). Preoperative PTPVS was inserted. Note that periportal collateral vessels (thin white arrows, D) all disappeared immediately after successful PTPVS (E). Preoperative 3-dimensional arterial reconstruction can be helpful when designing the surgical strategy. Portal vein stent can be identified with endoscopic biliary drainage tube. 
sized solid mass invading the distal CBD with upstream dilatation. EUS-fine needle aspiration was done and was reported poorly differentiated adenocarcinoma. An endoscopic retrograde bile drainage (ERBD) catheter was inserted.

Under the diagnosis of locally advanced pancreatic cancer (cT4N1, Stage III), the patient was directed to systemic chemotherapy and went through 16 cycles of FOLFIRINOX and combined chemoradiotherapy (CCRT) using Capecitabine +6000 cGy in 25 fractions in between the $12^{\text {th }}$ and $13^{\text {th }}$ cycle. While assessing resectability after neoadjuvant treatment, the cancer did not seem to have progressed and the disease status was stable. Preoperative last CA 19-9 and CEA level were noted to be $671 \mathrm{U} / \mathrm{ml}$ and $11.5 \mathrm{ng} / \mathrm{ml}$, respectively. The patient's general condition was quite acceptable. Surgical resection was considered as neither systemic metastasis nor disease progression were noted during neoadjuvant treatment. However, vascular invasions were still noted. Especially, a short segment of common hepatic artery (CHA)-gastroduodenal artery (GDA)-proper hepatic artery (PHA) confluence looked invaded and portal vein total obliteration with secondary periportal engorged collateral vessels was observed (Fig. 1A-C). Superior mesenteric artery (SMA) and proximal part of CHA were spared. In order to reduce intraoperative portal vein pressure and potential risk of severe intraoperative bleeding, mesenteric congestion, and hepatic ischemia, preoperative percutaneous transhepatic portal vein stent (PTPVS) was placed on March, $10^{\text {th }}$, 2020 (Fig. 1D-F).

\section{Operative findings}

On March, 25 ${ }^{\text {th }}$, 2020, exploratory laparotomy was performed. During the operation, severe adhesion around the pancreatic neck portion and celiac axis was observed. After careful dissection and isolation, total pancreatectomy with splenectomy and combined resection of common hepatic artery and SMV-SV-PV confluence with endend vascular reconstruction was performed (Fig. 2). Total pancreatectomy instead of PD with pancreatic head excision was done considering the severe atrophic change of the whole pancreas. The operation time was 7.5 hours with estimated blood loss of $1100 \mathrm{ml}$. No transfusion was given.

\section{Postoperative course and pathological examination}

The postoperative recovery was smooth without complications. Postoperative liver function test improved over time (Fig. 3). Follow-up blood CEA and CA 19-9 level on postoperative day seven were $2.55 \mathrm{ng} / \mathrm{ml}$ and 172.0 $\mathrm{U} / \mathrm{ml}$, respectively. The patient was discharged on the $8^{\text {th }}$ day after surgery under regular insulin therapy. It was reported that the residual tumor was of moderate differ-
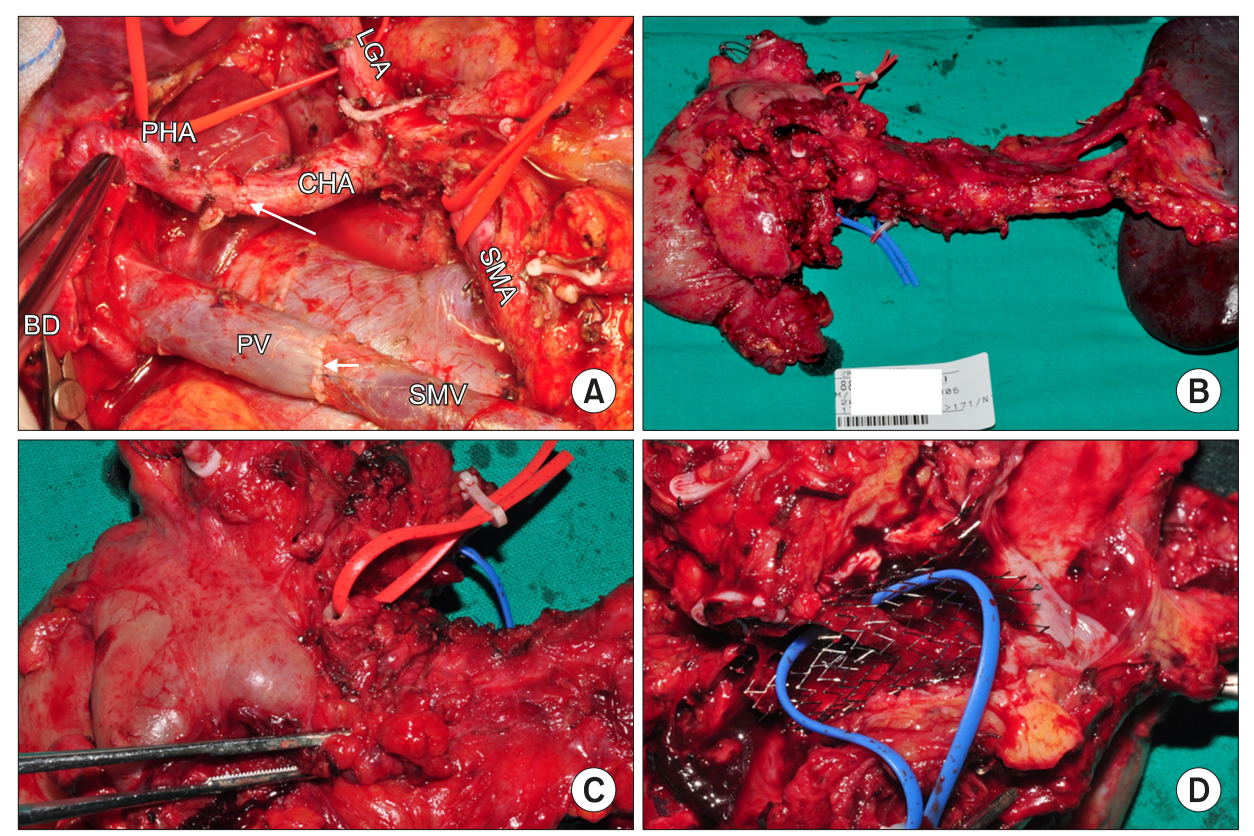

Fig. 2. Operative view and surgical specimens. Grossly marginnegative resection was performed. Note the arterial (long thin white arrow) and portal vein (short thin white arrow) reconstruction (A). Surgical specimen is excised en-bloc (B). Note the combined resection of common hepatic artery-gastroduodenal artery-proper hepatic artery confluence (red colored vessel-loop, C) and segment of PV with PTPVS (blue colored vessel-loop, D). 


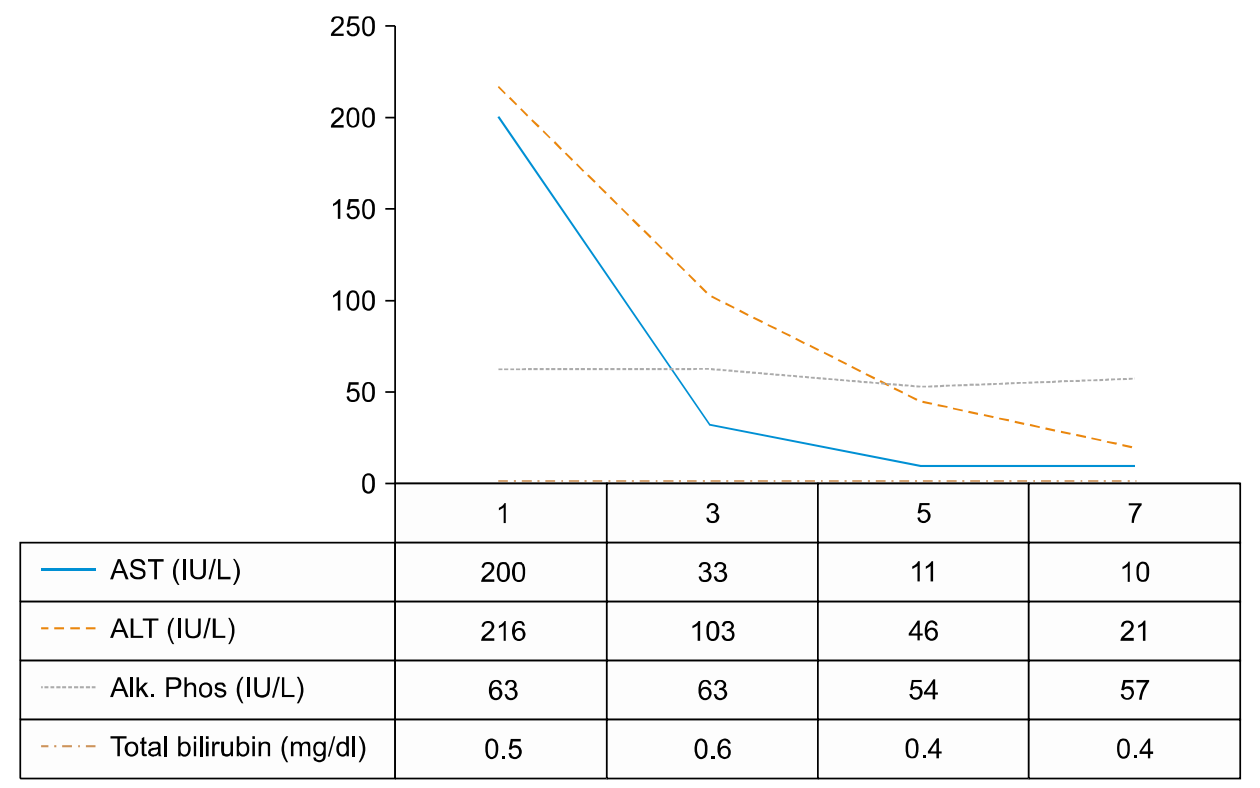

Fig. 3. Postoperative changes of liver function test. entiation with extracellular mucin formation, showing partial response to chemotherapy (Modified Ryan Scheme for tumor regression score 2). The size of the tumor was $3.5 \times 3.5 \times 2.2 \mathrm{~cm}(\mathrm{ypT} 2)$ with lymphovascular and perineural invasion. Excised SMV-SV-PV confluence showed invasion of carcinoma while hepatic artery was free of carcinoma. All resection margins were free from carcinoma. Two lymph nodes out of 12 showed metastatic carcinoma (ypN1).

After discharge, the patient came back for follow up visits during which he was treated for ascites and adjuvant chemotherapy is currently being planned at 6 week point of operation.

\section{DISCUSSION}

Percutaneous transhepatic portal vein stent (PTPVS) is usually applied to portal vein stenosis due to cancer recurrence, change after pancreaticobiliary cancer surgery, or liver transplantation. ${ }^{6-9}$ PTPVS is regarded as acceptable minimally invasive approach due to high success rate with low complications. ${ }^{10,11}$ Curative intent pancreatectomy may not be an indication when venous involvement is related to PV occlusion with periportal collaterals because there is significant risk of intraoperative bleeding and ischemic injury to the liver and bowels during the operation. In fact, in order to resolve these issues, Nakao et al. ${ }^{12}$ and Nakao and Takagi ${ }^{13}$ used antithrombogenic catheter bypass to reduce the PV pressure, and Bachellier et al. ${ }^{14}$ performed an intraoperative mesentericoportal shunt for pancreatic resection for LAPC with PV occlusion and portal hypertension, which all require additional specialized surgical instruments and vascular techniques leading to surgical complexity and prolongation of operation time.

The present case demonstrated preoperative PTPVS can play an important role in reducing intraoperative risk associated with PV occlusion in well selected pancreatic cancer. Considering that neoadjuvant chemotherapy became the standard in borderline and locally advanced pancreatic cancer, ${ }^{15}$ this strategy can be thought to have expanded the chance of curative pancreatectomy even in advanced pancreatic cancer, following neoadjuvant chemotherapy. Till now, only a few cases of preoperative PTPVS followed by pancreatectomy have been reported. ${ }^{16}$ Recently, Alden et al. ${ }^{17}$ reported PD following PTPVS in 15 patients with locally advanced pancreatic cancer (about $68 \%$ of resectability, 15 out of 22 patients) without perioperative mortality. In addition, survival differences were also observed between surgically resected group and non-resected group (17 months vs. 9 months, $p=0.0422$ ), suggesting this approach can be a potentially beneficial way for surgical resection in patients with PV, and SMV occlusion.

According to the current experience, followings are potential advantages of preoperative PTPVS in pancreatic cancer surgery. First, preoperative PTPVS makes surgical 
procedure simple without having to prepare for a complex intraoperative meso-systemic shunt. Second, the preoperative cavernous transformation around PV is diminished and can reduce the chance of intraoperative severe bleeding. Third, in spite of sequential disconnection of veins draining to SMV and PV, there was no small bowel congestion and hepatic ischemia due to maintenance of mesentero-hepatic flow by the PTPVS.

In the past, Fortner ${ }^{18}$ proposed a regional pancreatectomy for obtaining a wide tissue margin with regional lymphatic clearance in 1973, but pancreatectomy with extensive lymphadenectomy ${ }^{19}$ and combined vascular resection, especially, arterial resection have not been actively recommended in managing pancreatic cancer. ${ }^{20}$ Introduction of potent chemotherapeutic agents ${ }^{1,3,21}$ and advanced surgical techniques for vascular reconstruction thanks to liver transplantation are thought to be making a paradigm shift from conservative surgical approach to aggressive radical surgery again in well selected advanced pancreatic cancer. In addition, oncologic concept of total mesopancreas excision ${ }^{22}$ has been introduced to control local spread of pancreatic cancer. The oncologic significance of mesopancreas concept is still controversial, but it is expected to play a significant role for margin-negative resection in well-selected advanced pancreatic cancer following neoadjuvant treatment.

As Fortner" ${ }^{23}$ commented, "It is apparent that there is a very strong basis for the belief that regional pancreatectomies (=aggressive pancreatic cancer surgery, interpreted by authors) will result in significantly increased cure rates", It is true that pancreatic cancer survival is still dismal, however, long-term oncologic outcomes of resected pancreatic cancer will be optimistic in the near future due to integrated impact of perioperative multidisciplinary approach, potent chemotherapy, and advanced surgical technique for margin-negative resection. We never give up.

\section{CONFLICT OF INTEREST}

No potential conflict of interest relevant to this article was reported.

\section{ORCID}

Yoonwon Kook: https://orcid.org/0000-0002-0756-738X
Munseok Choi: https://orcid.org/0000-0002-9844-4747

Jung Yup Park: https://orcid.org/0000-0003-0110-8606

Yong Eun Chung: https://orcid.org/0000-0003-0811-9578

Man-Deuk Kim: https://orcid.org/0000-0002-3575-5847

Woo Jung Lee: https://orcid.org/0000-0001-9273-261X

Chang Moo Kang: https://orcid.org/0000-0002-5382-4658

\section{AUTHOR CONTRIBUTIONS}

Conceptualization: CMK, JYP, MDK. Data curation: YK, MC, CMK. Formal analysis: YK, MC, KYP, YEC, MDK, WJL, CMK. Funding acquisition: CMK. Methodology: CMK. Project administration: CMK. Visualization: CMK. Writing - original draft: YK, CMK. Writing - review \& editing: CMK.

\section{REFERENCES}

1. Conroy T, Hammel P, Hebbar M, Abdelghani MB, Wei AC-c, Raoul JL, et al. Unicancer GI PRODIGE 24/CCTG PA.6 trial: a multicenter international randomized phase III trial of adjuvant mFOLFIRINOX versus gemcitabine (gem) in patients with resected pancreatic ductal adenocarcinomas. J Clin Oncol 2018; 36(18 suppl):LBA4001.

2. Neoptolemos JP, Stocken DD, Friess H, Bassi C, Dunn JA, Hickey $\mathrm{H}$, et al. A randomized trial of chemoradiotherapy and chemotherapy after resection of pancreatic cancer. N Engl J Med 2004;350:1200-1210.

3. Oettle H, Neuhaus P, Hochhaus A, Hartmann JT, Gellert K, Ridwelski K, et al. Adjuvant chemotherapy with gemcitabine and long-term outcomes among patients with resected pancreatic cancer: the CONKO-001 randomized trial. JAMA 2013;310:14731481.

4. Oba A, Ho F, Bao QR, Al-Musawi MH, Schulick RD, Del Chiaro M. Neoadjuvant treatment in pancreatic cancer. Front Oncol 2020;10:245.

5. Kasumova GG, Conway WC, Tseng JF. The role of venous and arterial resection in pancreatic cancer surgery. Ann Surg Oncol 2018;25:51-58.

6. Wang JF, Zhai RY, Wei BJ, Li JJ, Jin WH, Dai DK, et al. Percutaneous intravascular stents for treatment of portal venous stenosis after liver transplantation: midterm results. Transplant Proc 2006;38:1461-1462.

7. Kim KR, Ko GY, Sung KB, Yoon HK. Percutaneous transhepatic stent placement in the management of portal venous stenosis after curative surgery for pancreatic and biliary neoplasms. AJR Am J Roentgenol 2011;196:W446-W450.

8. Zhou ZQ, Lee JH, Song KB, Hwang JW, Kim SC, Lee YJ, et al. Clinical usefulness of portal venous stent in hepatobiliary pancreatic cancers. ANZ J Surg 2014;84:346-352.

9. Ohgi K, Sugiura T, Yamamoto Y, Okamura Y, Ito T, Ashida $\mathrm{R}$, et al. Benign portal vein stenosis after pancreaticoduodenectomy. World J Surg 2019;43:2623-2630.

10. Park KB, Choo SW, Do YS, Shin SW, Cho SG, Choo IW. Percutaneous angioplasty of portal vein stenosis that complicates liver transplantation: the mid-term therapeutic results. Korean $\mathrm{J}$ 
Radiol 2005;6:161-166.

11. Jeon UB, Kim CW, Kim TU, Choo KS, Jang JY, Nam KJ, et al. Therapeutic efficacy and stent patency of transhepatic portal vein stenting after surgery. World J Gastroenterol 2016;22:98229828.

12. Nakao A, Nonami T, Harada A, Kasuga T, Takagi H. Portal vein resection with a new antithrombogenic catheter. Surgery 1990; 108:913-918.

13. Nakao A, Takagi H. Isolated pancreatectomy for pancreatic head carcinoma using catheter bypass of the portal vein. Hepatogastroenterology 1993;40:426-429.

14. Bachellier P, Rosso E, Fuchshuber P, Addeo P, David P, Oussoultzoglou E, et al. Use of a temporary intraoperative mesentericoportal shunt for pancreatic resection for locally advanced pancreatic cancer with portal vein occlusion and portal hypertension. Surgery 2014;155:449-456.

15. Scheufele F, Hartmann D, Friess H. Treatment of pancreatic cancer-neoadjuvant treatment in borderline resectable/locally advanced pancreatic cancer. Transl Gastroenterol Hepatol 2019;4: 32.

16. Scemama U, Birnbaum DJ, Ouaissi M, Turrini O, Moutardier V, Soussan J. Portal vein stent placement in five patients with chronic portal vein thrombosis prior to pancreatic surgery. J Vasc Interv Radiol 2016;27:889-894.
17. Alden D, Dudiy Y, Nassiri N, Friedland RJ, Amatulle P, Rosen RJ. Direct percutaneous transhepatic portomesenteric venous stenting in management of locally advanced pancreatic cancer. Am J Clin Oncol 2015;38:127-129.

18. Fortner JG. Regional resection of cancer of the pancreas: a new surgical approach. Surgery 1973;73:307-320.

19. Dasari BV, Pasquali S, Vohra RS, Smith AM, Taylor MA, Sutcliffe RP, et al. Extended versus standard lymphadenectomy for pancreatic head cancer: meta-analysis of randomized controlled trials. J Gastrointest Surg 2015;19:1725-1732.

20. Delpero JR, Sauvanet A. Vascular resection for pancreatic cancer: 2019 French recommendations based on a literature review from 2008 to 6-2019. Front Oncol 2020;10:40.

21. Neoptolemos JP, Palmer DH, Ghaneh P, Psarelli EE, Valle JW, Halloran CM, et al. Comparison of adjuvant gemcitabine and capecitabine with gemcitabine monotherapy in patients with resected pancreatic cancer (ESPAC-4): a multicentre, open-label, randomised, phase 3 trial. Lancet 2017;389:1011-1024.

22. Cao Z, Xu J, Shao Q, Zhang T, Zhao Y. Surgical treatment of pancreatic head cancer: concept revolutions and arguments. Chin J Cancer Res 2015;27:392-396.

23. Fortner JG. Surgical principles for pancreatic cancer: regional total and subtotal pancreatectomy. Cancer 1981;47(6 Suppl):17121718 . 